\title{
Entre Chenalhó y Chalchihuitán: (IN)MOVILIDAD FORZADA Y LÍMITES EN DISPUTA
}

\author{
Between Chenalhó y Chalchihuitán: \\ FORCED (IM)MOBILITY AND BORDER CONFLICT
}

\section{Carolina Pecker Madeo*}

DOI: https://doi.org/10.31644/ED.V7.N2.2020.A10

Resumen: El artículo aborda el desplazamiento forzado que movilizó a más de 5000 indígenas, en noviembre de 2017 en Los Altos de Chiapas, por motivos de conflictos limítrofes entre los municipios indígenas de lengua tsotsil, Chenalhó y Chalchihuitán. Para esto, presento sus antecedentes formales utilizando la información provista por documentos estatales y fuentes de prensa, así como también caracterizo la situación a partir de lo recabado en mapeos y entrevistas realizados en la zona de conflicto en colaboración con el traductor indígena de Médicos Sin Fronteras y con personal del Centro de Derechos Humanos Fray Bartolomé de Las Casas, en enero y febrero de 2018. Los resultados muestran que las prácticas de marcación limítrofe despliegan su conflictividad tanto en el ámbito jurídico formal como en la materialidad del territorio. A su vez, las entrevistas y mapeos permiten caracterizar las (in)movilidades forzadas y dar cuenta del modo en que derivaron en una situación de (des)territorialización precaria y crisis humanitaria.

* Universidad de Buenos Aires, Facultad de Filosofía y Letras, Instituto de Ciencias Antropológicas (ICA), Buenos Aires, Argentina. Líneas de investigación: violencia, desplazados internos y construcción de territorios en los Altos de Chiapas. Identificador ORCID: https:// orcid.org/0000-0001-9005-697X; Correo-e: pecker_madeo@hotmail.com.

Fecha de recepción: 22/01/2020. Fecha de aceptación: 30/04/2020. Fecha de publicación: 31/07/2020.

(cc) BY-NC-ND Páginas 280 - 307 
Palabras clave: conflicto limítrofe, desplazamiento forzado, municipios indígenas, (des)territorialización precaria

Abstract: The article aims to explain the forced displacement that mobilized more than 5000 indigenous people in November 2017 in the Highlands of Chiapas, due to border conflicts between the indigenous, tsotsil speaking, municipalities of Chenalhó and Chalchihuitán. I present its formal background using information provided by state documents and press sources, as well as characterize the situation based on what was collected in mappings and interviews conducted in collaboration with the indigenous translator of Doctors Without Borders in the conflict zone and with staff of the Fray Bartolomé de Las Casas Human Rights Center in January and February 2018. The results show that borderline dialing practices display their conflict both in the formal legal field as in the materiality of the territory. In turn, interviews and mappings allow characterization of forced (im)mobility and account for how they resulted in a situation of precarious (de)territorialization and a humanitarian crisis.

Keywords: border conflict, forced displacement, indigenous municipalities, precarious (de)territorialization

\section{Introducción}

El 13 de noviembre de 2017, el sonido de una amenazante balacera irrumpió en las montañas de los Altos de Chiapas. Miles de personas abandonaron sus hogares buscando resguardo, incluso allí donde tan sólo el relieve o la vegetación les permitiesen sentirse ocultas. Algunas decidieron mantenerse juntas o reagruparse e improvisar refugios cercanos a sus comunidades, esperando con ansias que la violencia terminara.

Más de 5000 personas fueron afectadas en este desplazamiento forzado, un fenómeno recurrente y vigente ${ }^{1}$ en México y en el estado. Si bien los

\footnotetext{
${ }^{1}$ De acuerdo con el registro histórico llevado a cabo por la Comisión Mexicana de Defensa y Promoción de los Derechos Humanos, A.C., el total de personas internamente desplazadas por la fuerza en México, hasta diciembre de 2018, es de 338,405. En 2018 el desplazamiento forzado interno masivo afectó a 11,491 personas en México y el estado de Chiapas concentró el 43.82\% del total de la población desplazada del país (CMDPDH, 2019: 13-15).
} 
desplazamientos en la región han estado en su mayoría vinculados a conflictos político-religiosos desde la década de 1970 - movilizando a cerca de 30.000 personas, en su mayoría indígenas tsotsiles provenientes del municipio de San Juan Chamula² (Martínez Velasco, 2005: 200)- y al conflicto armado interno desatado en enero de 1994 a partir del levantamiento del Ejército Zapatista de Liberación Nacional (EZLN) y las ofensivas militares y paramilitares (Chamberlin, 2013) -por el cual se estima que se han desplazado entre 50.000 y 84.0000 personas (Arana Cedeño y Del Riego, 2012: 34)-, la situación que atañe al presente artículo tiene la particularidad de vincularse con una disputa limítrofe entre dos municipios de lengua indígena, Chenalhó y Chalchihuitán ${ }^{3}$, ambos de población en su mayoría de habla tsotsil. ${ }^{4}$ Lo que distingue a las movilidades caracterizadas como "forzadas" de otro tipo de movimientos, como los viajes por turismo o de negocios, es que estas, impulsadas por la violencia, son llevadas a cabo con el fin de proteger la integridad física o enfrentar circunstancias adversas:

[...] nos referimos a las movilidades forzadas como resultado de situaciones de violencia que generan altos grados de inseguridad y temor. Es el caso de la delincuencia común, violencia intrafamiliar y de género, homofobia, exclusión, marginación, crimen organizado, narcotráfico y grupos paramilitares o de autodefensa; o también de abusos del propio Estado (Coraza de los Santos, 2018: 2).

En este sentido, como resultado de situaciones de violencia que generan altos grados de inseguridad y temor, el desplazamiento forzado interno es una forma específica de movilidad forzada cuya dimensión territorial no trasciende fronteras nacionales. Para referirme a este proceso y sus efectos en la territorialización ${ }^{5}$ de los sujetos desplazados, en este artículo recurro al término

\footnotetext{
${ }^{2}$ Este municipio concentró, junto con el municipio de Las Margaritas, el 57\% de los conflictos registrados en Chiapas entre los ańos 1960 y 2001 (Rivera Farfán, 2013: 92).

${ }^{3}$ Chenalhó, el cual con 36.111 habitantes posee una extensión territorial de $245,41 \mathrm{~km}^{2}$ y Chalchihuitán, municipio que cuenta con una población de 14.027 habitantes y un territorio de $183,21 \mathrm{~km}^{2}$ (INEGI, 2010).

${ }^{4}$ Forma castellanizada de tsots îl. Abarca un "conjunto de lenguas indígenas estrechamente relacionadas entre si" que "pertenecen al grupo cholano-tzeltalano de la familia lingüística maya” (Gobierno de México, 2020).

${ }^{5}$ El territorio implica una relación de apropiación socio-espacial que "se reproduce a lo largo 
de desterritorialización, ${ }^{6}$ no sólo en un sentido genérico de destrucción o abandono de territorios, sino también en el sentido de precarización territorial, en tanto efecto de la violencia sobre la territorialización de los sujetos, en situaciones de "efectiva inestabilidad o debilidad territorial". Las propiedades que evidencian la desterritorialización / territorialización precaria -a lo cual me refiero como (des)territorialización precaria- son:

la inestabilidad e inseguridad socioespacial; la fragilidad de lazos entre los grupos sociales y de estos con su espacio (tanto en términos de relaciones funcionales como simbólicas) y la movilidad sin dirección definida o la inmovilidad sin efectivo control territorial (Haesbaert, 2011: 274).

Con el objetivo de presentar los antecedentes del desplazamiento forzado y de caracterizar las (in) movilidades forzadas y sus efectos de (des)territorialización precaria y crisis humanitaria, ${ }^{7}$ realizo un análisis cualitativo basado en fuentes

de un continuum que va desde la dominación política-económica más "concreta” y "funcional" hasta la apropiación más subjetiva o "cultural-simbólica”. Aunque resulta equivocado escindir estas esferas, cada grupo social puede territorializarse, es decir, crear mediaciones espaciales que proporcionen un efectivo "poder" sobre su reproducción como grupos o individuos, a través de procesos de carácter más funcional o simbólico "dependiendo de la dinámica de poder y de las estrategias que están en juego” (Haesbaert, 2011:82-83).

6 "Desterritorialización, si es posible utilizar el concepto de manera coherente, nunca es 'total' o desvinculada de los procesos de re-territorialización, debe aplicarse a fenómenos de efectiva inestabilidad o debilidad territorial, sobre todo entre grupos socialmente más excluidos o profundamente segregados y, como tales, imposibilitados de ejercer un control efectivo sobre sus territorios" (Haesbaert, 2011: 258).

7 Una situación caracterizada como de crisis humanitaria refiere al conjunto de consecuencias derivadas de diversos factores políticos, económicos y socioculturales, o resultantes de desastres por eventos naturales, que: "además de cobrar vidas, también dan lugar a pérdidas de medios de vida e infraestructura, las cuales menoscaban la capacidad de subsistencia y recuperación de los sobrevivientes. La seguridad alimentaria se afecta por la destrucción de los medios de vida, sean cultivos, ganado, u otros medios de producción o generación de ingresos. Eventualmente pueden producirse situaciones de hambruna y grave afectación de las condiciones de salubridad por la falta de servicios básicos. Todo ello incide en la salud y puede llegar a cobrar más vidas en consecuencia. Cuando se trata de casos de emergencia compleja, es decir, debidos a la interrelación de diversos factores políticos, económicos y socioculturales que dan lugar a conflictos, hambrunas, y desplazamientos masivos, cuyos problemas provienen principalmente de la falta de acceso a las fuentes tradicionales de ingresos por períodos prolongados a causa de los desplazamientos y la inseguridad. En estas condiciones se agudizan las necesidades económicas para una subsistencia digna, dando lugar a la vulnerabilidad alimentaria, económica y de salud, aumentando la demanda de servicios de salud que se dificultan cuando hay contextos de 
secundarias y primarias, incluyendo documentos estatales, fuentes de prensa, entrevistas y mapeos realizados en la ciudad de San Cristóbal de Las Casas durante enero y febrero de 2018, en colaboración con Miguel López Girón, quien integró la brigada de ayuda humanitaria internacional de Médicos Sin Fronteras (MSF), como traductor indígena en la zona del conflicto, y Guadalupe Moshan, trabajadora del Centro de Derechos Humanos Fray Bartolomé de Las Casas (CDHFBC) e integrante del Equipo Zona Altos. ${ }^{8}$

Imagen 1: Médicos Sin Fronteras en Chalchihuitán, fotografía por Miriam de León

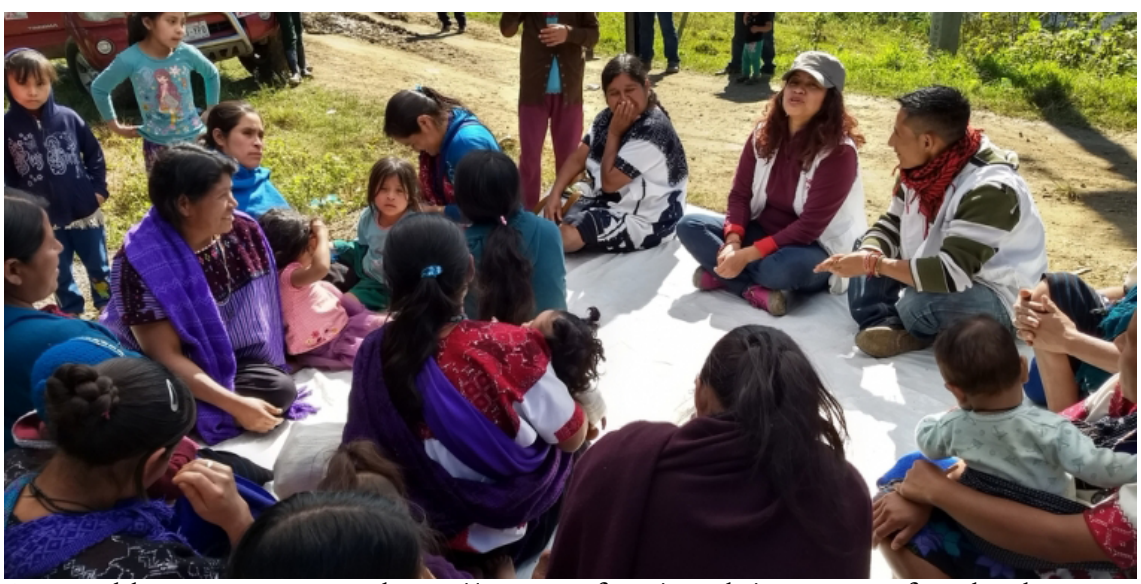

Disponible en: https://www.msf.mx/article/mexico-msf-se-despliega-enchalchihuitan-chiapas-para-atender-a-los-desplazados-en-la-zona (Consultado el 29 de septiembre de 2018)

\section{Los límites en disputa}

Los antecedentes de este conflicto se remontan a la década de 1970, cuando, en el contexto de la Ley Federal de la Reforma Agraria (1971), los municipios de Chenalhó y Chalchihuitán buscaron el reconocimiento y la titulación de sus tierras comunales por parte del sistema jurídico dominante. Con esta ley se

violencia” ONU. Sistema de Las Naciones Unidas en Perú, Disponible en: < http://onu.org.pe/ temas/desastres-y-crisis-humanitarias/> [Consultado 14 de abril de 2020].

8 "Es el equipo encargado de acompañar procesos sociales de defensa en la región conocida como Los Altos de Chiapas, así como otros municipios colindantes con esta región, se caracteriza por ser una zona mayoritariamente indígena de los pueblos tsotsiles, tseltales, además de la población mestiza y del extranjero que se ha asentado en esta región” (CDHFBC, 2019). 
reglamentaban las disposiciones agrarias del artículo 27 constitucional (antes de su reforma de 1992), incluyendo aquellas tendientes al Reconocimiento y Titulación de Bienes Comunales que las comunidades indígenas poseían "desde tiempo inmemorial". Cabe aclarar que, en contraste con las acciones agrarias, aplicadas a los ejidos -dotaciones, ampliaciones e incorporación de tierras al régimen ejidal-, aquellas destinadas a las comunidades indígenas han tenido el carácter de reconocimientos, restituciones y titulaciones, y han sido menos frecuentes (Jiménez Huerta y Ayala, 2015: 115).

Las propiedades sobre los bienes comunales de Chenalhó y Chalchihuitán fueron, entonces, reconocidas por la Secretaría de Reforma Agraria9 e inscriptas en el Registro Público de la Propiedad y en el Registro Agrario Nacional. Mediante la resolución presidencial de Reconocimiento de Tierras de Bienes Comunales, publicada en Diario Oficial de la Federación (DOF) el día 29 de agosto de 1975, "se reconoce y titula correctamente a favor de poblado Chalchihuitán, Municipio de Chalchihuitán, del Estado de Chiapas, una superficie total de 17.948 [...] Diecisiete mil novecientas cuarenta y ocho hectáreas". En la ejecutoria se reconocieron 17.696 hectáreas y luego, en 1981, se agregaron 252 hectáreas ${ }^{10}$ (Décimo Tercer Tribunal Colegiado en Materia Administrativa del Primer Circuito, 2005:12.). Mientras que la resolución presidencial de Chenalhó del 6 de octubre del mismo ańo reconoce y titula un total de 15.625 hectáreas. La misma se ejecutó en el año de 1976 con 14.438 hectáreas (Reyes Ramos, 2004:20).

Así, las resoluciones presidenciales mencionadas (DOF, 1975a:16; DOF, 1975b:17) aseveran:

[...] dicha comunidad comprobó fehacientemente estar en posesión de sus terrenos en forma pacífica, pública y continua desde tiempo inmemorial [...] la comunidad de que se trata, no tiene conflictos por límites con los poblados circunvecinos de acuerdo con las actas levantadas al efecto y las opiniones del Instituto Nacional Indigenista y de la Dirección General de Bienes Comunales

\footnotetext{
${ }^{9}$ El 17 de octubre de 2017, el Pleno Cameral aprobó cambios al artículo 51 de la Ley General de Desarrollo Social para sustituir la denominación de "Secretaría de la Reforma Agraria" por la de "Secretaría de Desarrollo Agrario, Territorial y Urbano" (Cámara de Diputados de la Unión, 2017).

${ }^{10}$ Acta de Posesión y Deslinde, 1 diciembre 1980; Acta de Posesión y Deslinde Complementaria, 19 octubre 1981 (Décimo Tercer Tribunal Colegiado en Materia Administrativa del Primer Circuito, 14 enero 2005:12).
} 
son en el sentido de que es procedente el reconocimiento y titulación de los terrenos comunales del poblado mencionado, por haberse reunido los requisitos que señalan la Ley Federal de Reforma Agraria y el Reglamento respectivo (DOF, 1975a:16; DOF, 1975b:17).

Si bien se supone que ambas comunidades comprobaron "fehacientemente estar en posesión de sus terrenos en forma pacífica, pública y continua desde tiempo inmemorial", paradójicamente, este mismo proceso de inscripción formal de sus propiedades va a marcar el comienzo de las disputas agrarias públicas y oficiales entre los municipios de Chalchihuitán y Chenalhó. Las mismas surgen a partir de diferencias en las "colindancias y linderos" señaladas por las resoluciones presidenciales mencionadas: en la correspondiente al municipio de Chenalhó se indica "la colindancia natural (río de por medio) con los terrenos comunales de San Pablo Chalchihuitán”, mientras que en la resolución del último municipio esto no es mencionado. A continuación, señalo cuál sería la "colindancia natural" que indica la resolución de Chenalhó.

En esta imagen satelital (Imagen II) cada municipio fue resaltado con colores Imagen II: "Límites naturales entre los municipios de Chenalhó, Chalchihuitán y Pantelho"

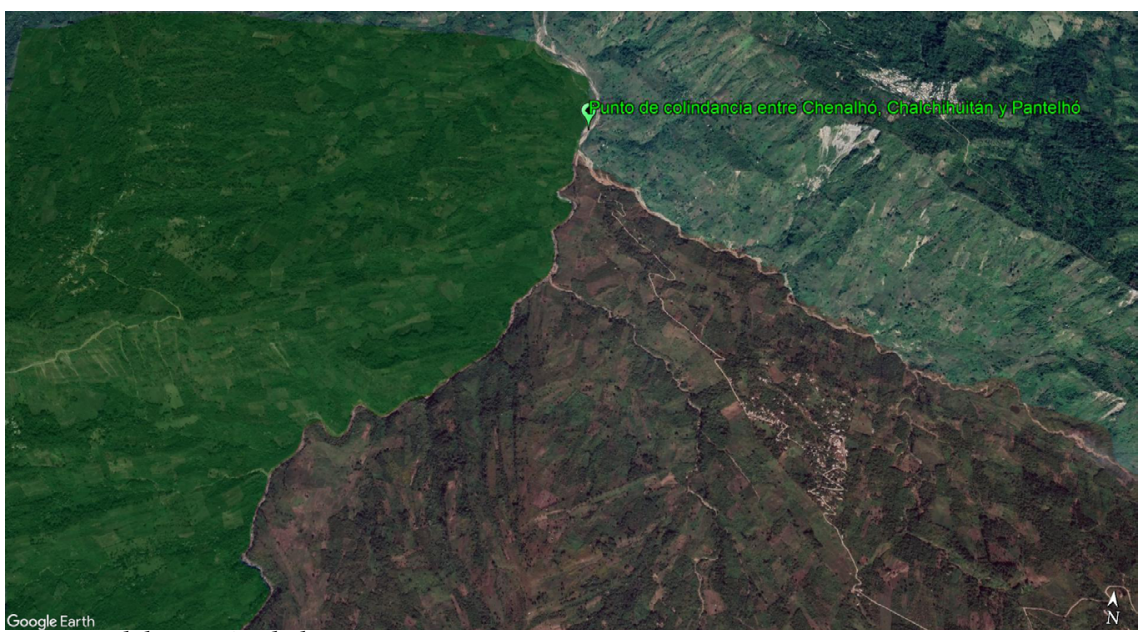

Fuente: Elaboración de la autora, 2019.

diferentes. A la izquierda, en color verde oscuro, se encuentra el municipio de Chalchihuitán; abajo y al centro, en color rojizo, el de Chenalhó y arriba a la 
derecha el de Pantelhó. Se marca también el punto de colindancia entre los tres municipios sobre el río Mashiló, a partir de allí, el río se bifurca. Hacia el sureste, marcando el límite entre Chenalhó y Pantelhó. Y hacia el suroeste, señalando el límite entre Chenalhó y Chalchihuitán. Estos "límites naturales" son los que figuran también en los mapas oficiales de la región. Sin embargo, si consideramos la resolución presidencial del año 1975, en lugar de seguir el curso del río habría que trazar un límite "artificial", que podemos imaginar desde el punto de colindancia señalado en línea recta hacia el sur, lo cual implicaría reconocer una mayor cantidad de hectáreas como pertenecientes a Chalchihuitán.

Pese a esta diferencia entre los límites indicados en las resoluciones, ambas fueron aprobadas y publicadas en el Diario Oficial de la Federación. Al percatarse de esta situación, y como intento de frenar la conflictividad, el 6 de agosto de 1976 ambos municipios firmaron un acuerdo con la intervención de las autoridades del Gobierno del Estado y la Secretaría de la Reforma Agraria, comprometiéndose a respetar la colindancia natural del río. Sin embargo, este acuerdo fue desestimado por Chalchihuitán.

Se firmó un acuerdo de respeto y reconocimiento de las mojoneras que por tiempo inmemorial se han considerado como límites, en el que se señaló por ambas comunidades, los puntos principales que marcan el rumbo que como límite natural es el río, con los nombres de Balumpi, Chixtal y Mashiló, convenio que, fue respetado durante cinco años, los habitantes de Chalchihuitán, en mil novecientos ochenta y uno, no tenían porqué construir muros y alambrar tierras que tienen en posesión, los habitantes de Chenalhó; que esta acción provocó que en el año de mil novecientos ochenta y tres, los habitantes de Chenalhó se organizaron y levantaron el alambrado y destruyeron los muros (Décimo Tercer Tribunal Colegiado en Materia Administrativa del Primer Circuito, 2005:23-24) ${ }^{11}$.

Las autoridades municipales de Chenalhó denuncian que, cinco años después de haberse firmado el acuerdo, habitantes de Chalchihuitán comenzaron a construir muros y alambrar tierras, tratando de fijar en el territorio las colindancias señaladas en su resolución presidencial del año 1975, las cuales, ${ }^{11}$ Décimo Tercer Tribunal Colegiado en Materia Administrativa del Primer Circuito, Solicitud de Ejercicio de la Facultad de Atracción 19/2004-SS, 14 enero 2005:23-24. 
según Chenalhó, son erróneas ya que no respetan "las mojoneras que por tiempo inmemorial se han considerado como límites" (ibídem).

Ocurre entonces que esta disputa, que se va desarrollando en el ámbito judicial, en un plano escrito y formal, se despliega también en el territorio, donde las personas buscan (des)marcar los límites mencionados, pues en su definición está en juego el acceso a sitios considerados sagrados, fuentes de agua y parcelas cultivables (Sheseña, 2014), ${ }^{12}$ considerando además que las mismas se encuentran entre municipios cuya dedicación es principalmente agrícola, ${ }^{13}$ por lo que la conflictividad en torno a la tierra adquiere una relevancia de carácter fundamental. Este forcejeo se pone "en pausa" hasta el 20 de agosto del ańo 2001, cuando los habitantes de Chalchihuitán son nuevamente denunciados por abrir una brecha en tierras donde vive gente de Chenalhó "destruyendo cosechas como son: milpas, cafetales, cañaverales, naranjales, platanales y las hortalizas que se han cultivado para el sustento familiar" (idem) y, el 3 de abril de 2002, por volver a construir muros de concreto "que servirán como mojoneras; y el veintisiete de ese mes y año penetraron nuevamente a sus parcelas, para destruir y cosechar el producto de su trabajo; asimismo, que se quedaron para cultivar sus tierras" (idem). En relación con estas prácticas, todos los muros mojoneros identificados en la zona fueron construidos por comuneros de Chalchihuitán, mientras que no pudieron hallarse mojones instalados por comuneros de Chenalhó, "excepto los mojones naturales, por ellos seńalados, que en algunos segmentos es el cauce del río" (Grupo Multidisciplinario, 2010: 37).

\footnotetext{
${ }^{12}$ Hay que tener en cuenta que la tierra correspondiente a la franja disputada adquiere un nuevo valor a partir de los ańos setenta, cuando la producción de café crece en el altiplano chiapaneco, lo cual ha alentado la conflictividad. A este aumento del interés comercial sobre la tierra, en un contexto en el cual se ha agotado la frontera agrícola hacia la cual crecer (y ante la cancelación del reparto agrario desde la reforma al Artículo 27 constitucional en el año 1992), se le suma la presión por la demanda de tierra fértil derivada de formas de herencia y de organización territorial locales, cuyos límites ponen fronteras para que personas fuera del grupo parental no se apropien de los terrenos considerados propios, al mismo tiempo que "pone los límites para la expansión hacia fuera (excepto de las tierras hoy en disputa)" (Grupo Multidisciplinario, 2010: 127-128).

${ }^{13}$ Según los Planes Municipales PNUD (Plan Municipal para la Reducción de Vulnerabilidades y Riesgos, elaborados en conjunto con el Programa de las Naciones Unidas para el Desarrollo en el ańo 2016), en ambos municipios la principal actividad productiva es la agricultura destinada al cultivo de maíz y frijol para el autoconsumo. La segunda actividad económica es la producción de café, mientras que los huertos frutales ocupan el tercer lugar en los cultivos del territorio.
} 
A lo largo del conflicto se suceden denuncias y juicios agrarios, durante los cuales las autoridades de cada municipio recurren a diferentes tipos de documentos para intentar legitimar su propiedad territorial y denunciar la "invasión" de los otros. Así, mientras desde Chalchihuitán se basan en la resolución presidencial del 29 de agosto de 1975 y sus correspondientes actas de posesión y deslinde (Poder Judicial del Estado de Chiapas, 2017), desestimando el valor legal del acuerdo del año $1976^{14}$ las autoridades de Chenalhó argumentan que debe respetarse la colindancia natural del río, de tiempo inmemorial, y consideran legítimo el acuerdo mencionado entre los municipios.

Según consta en uno de los juicios agrarios, Chalchihuitán demandó la restitución de 58 hectáreas (TUA del Distrito Núm. 3, 2000) ante lo cual San Pedro Chenalhó (TUA del Distrito Núm. 3, 2002) demandó la nulidad de la resolución presidencial que había reconocido y titulado los bienes comunales de San Pablo Chalchihuitán, bajo el argumento de que "se sobrepuso una superficie de 250-00-00 hectáreas (doscientas cincuenta hectáreas), que históricamente han venido poseyendo desde tiempo inmemorable" (Décimo Tercer Tribunal Colegiado en Materia Administrativa del Primer Circuito, 2005). Ante la sentencia a favor de Chenalhó, el 6 de julio de 2003, por parte de Magistrado del Tribunal Unitario Agrario (TUA), Chalchihuitán presenta un recurso de revisión, cuya resolución fue emitida el 9 de octubre de 2003. Al no verse favorecido, el 10 de junio de 2004, el mismo municipio interpone un Amparo Directo ante el Décimo Tribunal Colegiado en Materia Administrativa (39/2004-550). La sentencia, dictada el 6 de mayo de 2005, concede el amparo a Chalchihuitán. A partir de ello, Chenalhó "demanda la nulidad de la ejecución de San Pablo Chalchihuitán radicándose el juicio número 781/05, del índice del Tribunal Unitario Agrario del Tercer Distrito"15.

Hacia el mes de enero del año 2008, con la disputa aún sin resolverse, comuneros de ambos municipios comienzan a limpiar y preparar las parcelas

\footnotetext{
${ }^{14}$ Sostienen que este acuerdo "No es legal porque, en su concepto, el Comisariado de bienes comunales del poblado Chalchihuitán que lo firmó carece de facultades para celebrarlo; además de que aducen que no fue aprobado por la asamblea de comuneros" (Décimo Tercer Tribunal Colegiado en Materia Administrativa del Primer Circuito. Solicitud de Ejercicio de la Facultad de Atracción 19/2004-SS, 14 enero 2005:21).

${ }^{15}$ Referenciado en el punto de acuerdo del 3 de marzo de 2009 la Cámara de Diputados federal en que se exhorta a la Secretaría de la Reforma Agraria a continuar atendiendo el conflicto agrario entre Chenalhó y Chalchihuitán en Chiapas.
} 
para cultivar dentro de lo que consideran como sus límites agrarios (Grupo Multidisciplinario, 2010: 27). Esto agudiza el conflicto y resulta en el asesinato de Antonio Jiménez Ruiz, un indígena tsotsil originario de la comunidad de Majomut, municipio de Chenalhó. La responsabilidad del crimen, ocurrido el 7 de abril de 2008, es adjudicada a habitantes de Chalchihuitán (Henríquez, 2008).

Ante el aumento de la violencia, los gobiernos del estado y federal retoman el caso y establecen mecanismos de negociación. ${ }^{16}$ Como parte de esta estrategia de atención, ratificada por ambos municipios, un Grupo Multidisciplinario inicia su funcionamiento a partir del 15 de agosto de 2008, con el objetivo de estudiar la situación y proponer una resolución. El informe resultante adjudica la responsabilidad del conflicto al mal desempeño de la Secretaría de la Reforma Agraria (SRA) e indica, entre otras sugerencias, que

El Estado mexicano debe compensar el sufrimiento social que Chalchihuitán y también Chenalhó han vivido, por haberlos enfrascado en un conflicto en el que ambos han perdido tiempo, energía y dinero; por lo que la SRA debe reparar el daño y dignificar a los pueblos. Finalmente, la mesa de diálogo entre el gobierno, Chenalhó y Chalchihuitán, debe reconocer los dos derechos. Por un lado, reconocer que "el error de los topógrafos" como principal causante del conflicto, debe ser jurídicamente enmendado, mediante los procedimientos que sean necesarios, y regresar el límite a los que marcan sus títulos primordiales, reconociendo el justo valor de los mismos. Al mismo tiempo, la SRA debe reconocer los derechos agrarios adicionales, creados a favor de Chalchihuitán, y buscar medidas compensatorias que entreguen tierras de

${ }^{16}$ Definen entonces "una estrategia de atención que se concreta en los documentos "Reglas de Operación del Comité Técnico para la Atención del Conflicto de Límites entre las Comunidades de San Pedro Chenalhó y San Pablo Chalchihuitán” y "Estrategias de Atención San Pablo Chalchihuitán y San Pedro Chenalhó", que se firman el 24 de junio de 2008 en las instalaciones de la Comisión para el Desarrollo de los Pueblos Indígenas (CDI), en San Cristóbal de Las Casas, con la presencia del secretario de Gobierno de Chiapas y el delegado federal de la Secretaría de la Reforma Agraria. Para dar certeza a los pueblos, esta estrategia de atención fue ratificada por los pueblos ante el gobernador del estado en palacio de gobierno, en la ciudad de Tuxtla Gutiérrez, el 28 de julio de 2008 y firman nuevamente los documentos titulados "Firma de compromisos de los Pueblos San Pedro Chenalhó, San Pablo Chalchihuitán, Gobierno Federal y Gobierno del Estado, para buscar solución al Conflicto Territorial entre los municipios Chenalhó y Chalchihuitán, para mantener la Paz, la Tranquilidad y el Estado de Derecho"” (Grupo Multidisciplinario, 2010: 27-28). 
igual calidad a los parcelarios de Chalchihuitán que resulten afectados por la devolución de esos terrenos a manos de los bienes comunales de Chenalhó (Grupo Multidisciplinario, 2010: 139).

Sin embargo, en el año 2014, el Gobierno del Estado y la Secretaría de Desarrollo Agrario, Territorial y Urbano (SEDATU), sin reconocer el informe del grupo multidisciplinario, señala que quienes deben recibir la compensación a cambio de las tierras son los habitantes de Chenalhó (Santiago Rodríguez, 2017: 2).

En este contexto de contradicciones e incertidumbre, pobladores de Chenalhó destruyen alambrados y muros de concreto (Henríquez, 2014) que los comuneros de Chalchihuitán habían construido durante el año 2013 para demarcar lo que consideraban sus límites territoriales (García Núñez y Cruz Rueda, 2019: 33). Aumentan las denuncias acerca del accionar de grupos armados o paramilitares, a la vez que los enfrentamientos encrudecen hasta provocar el desplazamiento forzado de 500 personas (Avendaño, 2014). Una vez más, habitantes de ambos municipios denuncian "invasiones" y exigen la intervención de los Tribunales Agrarios.

El 3 de julio de 2015 es asesinado Salvador Luna, de 16 años, originario de la comunidad de Chenmut en Chalchihuitán. Como su homicidio es adjudicado a habitantes de Majopepentik, comunidad vecina pero que pertenece a Chenalhó, alrededor de 300 indígenas se desplazan ante el temor de ser agredidos (Henríquez, 2015). Unos meses después, el 23 de septiembre, autoridades de ambos municipios firman un "Convenio de Paz"17 con la presencia del gobernador Manuel Velasco Coello y del Secretario General estatal Juan Carlos Gómez Aranda, en el cual se comprometen a respetar el futuro fallo del Tribunal Unitario Agrario y a no recurrir a ningún recurso legal o de otra índole en la superficie en cuestión y en las colindantes a sus comunidades.

\footnotetext{
${ }^{17}$ Para llevar a cabo el Convenio de Paz y Solución Definitiva al Conflicto Social Agrario fueron convocados "El gobierno Estatal C. Manuel Velasco Coello, secretario general estatal Lic. Juan Carlos Gómez Aranda; en el poder judicial, el Magistrado Presidente del Tribunal Superior de Justicia y del Consejo de la Judicatura del Estado Lic. Rutilio Cruz Escandón Cadenas, en el poder legislativo el diputado Jorge Enrique Hernández Berma por parte de estas autoridades estatales; las autoridades agrarias así como los H. Ayuntamientos de ambos municipios indígenas de Chalchihuitán y Chenalhó” (García Núñez y Cruz Rueda, 2019: 31-32).
} 
Mientras esto sucedía, grupos paramilitares ${ }^{18}$ de Chenalhó detonaban armas de alto calibre en la franja de conflicto, desplazando a los comuneros de Chalchihuitán, aunque todavía de un modo "temporal y de bajo impacto por el nivel de violencia y de fuerza empleada" (García Núñez y Cruz Rueda, 2019: 32). Dos años más tarde, la marcación territorial por medio de prácticas de violencia -balaceras, destrucción de casas y amenazas- se intensifica.

Samuel Pérez Luna, poblador de Kanalumtik, Chalchuihuitán, resulta asesinado el 18 de octubre de 2017. Unas semanas más tarde, entre el 12 y el 13 de noviembre, grupos armados de corte paramilitar (CDHFBC, 2018) provocan el desplazamiento forzado de casi 6 mil indígenas tsotsiles, 5023 de Chalchihuitán y 950 de Chenalhó (CIDH, 2018) quienes tuvieron que refugiarse en bosques y montañas ante el temor de perder sus vidas.

El 13 de diciembre de 2017 el TUA emite su resolución a favor del municipio de Chenalhó, invalidando la delimitación de las fronteras realizada por la Secretaría de la Reforma Agraria, y ordena que la Secretaría de Desarrollo Agrario, Territorial y Urbano (SEDATU) vuelva a delimitar la superficie en conflicto, 364 hectáreas (SEGOB 2018: 66), que deberán ser restituidas a dicho municipio, incluyendo además "la entrega de 15 millones de pesos al municipio perdedor (Chalchihuitán), la construcción de 300 viviendas a cargo de la SEDATU y la puesta en marcha de proyectos productivos para todos los habitantes, entre otras acciones" (Henríquez, 2017).

Sin embargo, pese al fallo del Tribunal Unitario Agrario y los acuerdos celebrados entre autoridades municipales, las personas desplazadas de Chalchihuitán denuncian que su situación continúa sin resolverse.

\section{Campamentos y caminos forzados}

Conforme a los Principios Rectores sobre el desplazamiento de personas en el interior de su propio país -aprobados por la Organización de Naciones Unidas en el año 1998-, en el Artículo No3 de la Ley para la prevención y atención del desplazamiento interno en el Estado de Chiapas se define a los desplazados internos como:

personas o grupos de personas asentadas en el Estado de Chiapas que se han visto forzadas u obligadas a abandonar, escapar o huir de su lugar de residencia

\footnotetext{
${ }^{18}$ Grupos que operan en la zona de conflicto con armas de uso exclusivo del Ejército (García Núñez y Cruz Rueda, 2019: 40).
} 
habitual, en particular como resultado o para evitar los efectos de un conflicto armado, de situaciones de violencia generalizada, de violaciones de los derechos humanos o de catástrofes naturales o provocadas por el ser humano y que no han cruzado los límites territoriales del Estado (Periódico Oficial del Estado de Chiapas, 2012).

El desplazamiento forzado implica, entonces, un evento dramático de escape o huida, cuya movilidad no traspasa las fronteras del mismo Estado, pero abarca un proceso que lo trasciende, ya que sus efectos perduran tras el arribo a los "nuevos" territorios.

Para referirme a este proceso y sus efectos, me propongo caracterizar las (in)movilidades forzadas que se han puesto en práctica en el marco del conflicto limítrofe entre los municipios de lengua indígena de Chenalhó y Chalchihuitán, con el objetivo de dar cuenta del modo en que derivaron en una situación de (des)territorialización precaria y crisis humanitaria. Presento para ello mapeos de los campamentos realizados a dos meses de haberse producido el desplazamiento forzado y a un mes de haberse emitido el fallo del Tribunal Agrario, momento en el cual alrededor de 4000 personas ya habían retornado a sus hogares y permanecían en los campamentos unas 1000 (MSF, 2018; CDHFBC, 2018). ${ }^{19}$ Cabe aclarar que las referencias en los mapas son aproximadas, pues su utilización en el artículo tiene como objetivo permitirle al lector figurarse las dimensiones territoriales del conflicto, a la vez que su proceso de elaboración favoreció el diálogo y la reflexión con mis interlocutores en el campo.

Miguel López Girón, quien se encontraba trabajando como traductor indígena para una organización de ayuda humanitaria en la zona de conflicto, se reunió conmigo durante sus retornos provisionales en la ciudad de San Cristóbal de Las Casas. En sus estadías en Chalchihuitán, a lo largo del mes de enero de 2018, Miguel se alojó con la brigada de Médicos Sin Fronteras $(\mathrm{MSF})^{20}$ en la parroquia San Pablo, ubicada en la cabecera municipal.

\footnotetext{
${ }^{19} \mathrm{Al}$ día 4 de enero de 2018 las cifras estimadas son de 3858 personas que retornaron a sus comunidades y 1165 personas que no (CDHFBC, 2018).

${ }^{20}$ Médicos Sin Fronteras (sin fecha) es una organización de acción médico-humanitaria sin fines de lucro que asiste a personas amenazadas por "conflictos armados, violencia, epidemias o enfermedades olvidadas, desastres naturales y exclusión de la atención médica” Disponible en: $<$ https:/www.msf.org.ar/conocenos/quienes-somos> [Consultado 20 de diciembre de 2019].
} 
Cuenta que allí “el padre Sebastián” los invitó a quedarse en la parroquia y les ofreció varias comidas. Este ha sido el lugar desde donde salían hacia los campamentos y al cual retornaban por las tardes: "Nosotros llegamos aquí al centro, está el [sic] parroquia, y de ahí, este, agarramos este camino”, con esta frase Miguel comenzó a relatar sus recorridos por Chalchihuitán, tomando siempre la parroquia como referencia. A continuación, señalo la ubicación de la parroquia San Pablo en la cabecera de Chalchihuitán y los caminos que desde ahí tomaron hacia los campamentos de desplazados.

Imagen III: "Chalchihuitán: Parroquia San Pablo, caminos y 9 campamentos"

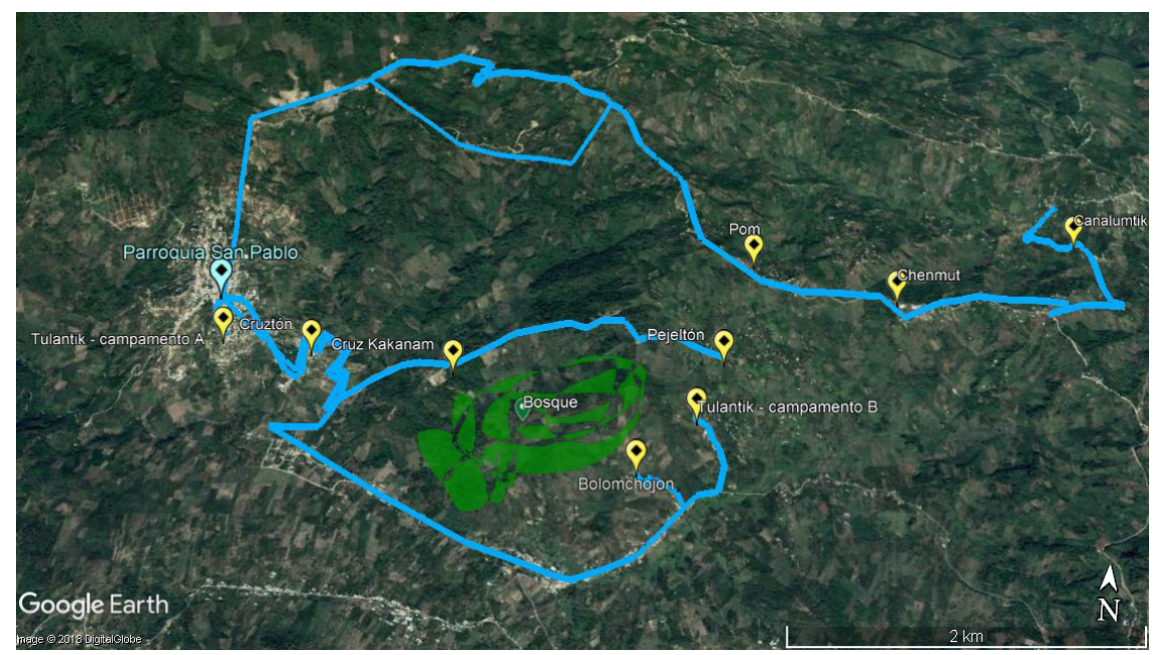

Fuente: Elaboración de la autora y Miguel López Girón, 2019.

Los primeros campamentos informales o refugios auto-establecidos ${ }^{21}$ se fueron conformando a partir de los días 12 y 13 de noviembre, cuando las y los habitantes de las comunidades próximas al límite municipal comenzaron a escapar de sus casas.

Entre bloqueos de caminos y agresiones de grupos armados, algunas personas lograron llegar a la cabecera municipal de Chalchihuitán o a casas de amigos y familiares, mientras que otras huyeron hacia los bosques en las montañas: "Decían que intentaron salir, pero cuando vieron que estaba

\footnotetext{
21 "Los primeros espacios donde se va a vivir el refugio auto-establecido son los intersticios, los terrenos baldíos, los bosques (o fragmentos de bosque en las zonas urbanas), los muelles" (Agier, 2012:491).
} 
cortado y que estaba el grupo armado ahí, entonces se fueron a seguirle en la montaña" (Entrevista a Guadalupe Moshan, San Cristóbal de Las Casas, 5 de febrero de 2018).

Miguel me señala una las zonas en donde algunas personas permanecieron escondidas durante alrededor de dos semanas -ver "bosque" en Imagen III- y me cuenta que incluso hubo mujeres que no tuvieron más opción que dar a luz a sus hijos allí, en la intemperie (Entrevista a Miguel López Girón, San Cristóbal de Las Casas, 5 de febrero de 2018).

Pasados algunos días, el presidente municipal les solicitó a quienes habían llegado a la cabecera que regresaran a sus comunidades (Entrevista a Guadalupe Moshan, San Cristóbal de Las Casas, 7 de febrero de 2018). Sin embargo, ante la continuidad de los ataques armados, las personas desplazadas no volvieron a sus casas. En algunos casos, ocuparon canchas de deportes y templos como puntos de reunión o de refugio durante el día, para luego, por la noche, subir a la montańa y protegerse de posibles agresiones (SADEC, 2017: 1). Hubo quienes improvisaron tolderías con los materiales que encontraron a su alcance o con los que luego recibieron en carácter de donaciones. La mayoría de los campamentos se construyeron con bolsas de nylon o láminas de plástico a modo de paredes y techos. Al respecto, dice Miguel, enfatizando la precariedad de los mismos: "Es totalmente plástico sí... ellos viven en una casa de plástico, o sea, no tan casa pues, es plástico y pues es un campamento de tierra, no tiene nada de casa" (Entrevista a Miguel López Girón, San Cristóbal de Las Casas, 30 de enero de 2018).

Según el informe de la Comisión Interamericana de Derechos Humanos $(\mathrm{CIDH}, 2018)$ en Chalchihuitán se registraron los siguientes números estimados de personas desplazadas por comunidad: Ch'en Mut: 700 personas, Pom: más de 2000 personas, Tozomolton: más de 800 personas, C'analumtic: 600 personas, Bejelton: 150 personas, Tulantic: 350 personas, Bolomchojon: 150 personas, Cruz CaCanam: 200 personas, Cruzton: 73 personas. ${ }^{22}$ De estas comunidades, ubicadas en la zona del conflicto

22 También se mencionan las siguientes comunidades afectadas por la situación general de violencia: Balunac'o, Cotolchij, Lobolaltic, Pacanam, Pat C'anteal, Chiquin Xulum, y Saclum. En Chenalhó hubo varias comunidades afectadas en las cuales las personas salían por las noches a refugiarse: La Limas (más de 900 habitantes), Campo Los Toros (30 habitantes), Vayem y Vacax (4 familias), Primera fracción de Yabteclum (700 habitantes), Tsabalho, Majompepentik (más de 800 personas), Fracción Polho (en donde habría 150 personas provenientes de Majompepentik) (CIDH, 2018). También se contabilizaron 100 personas de la comunidad de 
en Chalchihuitán, Miguel recuerda haber visitado nueve campamentos, correspondientes a ocho comunidades. Cabe aclarar que de las anteriores, por un lado, no mencionó a la comunidad de Tzomoltón y que, por el otro, señaló dos campamentos conformados por habitantes de Tulantik, los cuales corresponden a dos momentos diferentes, ("Tulantink - campamento A" y "Tulantink - campamento B" en la Imagen III), dado que estas personas primero se refugiaron en la cabecera municipal y luego se movieron cerca de su comunidad (Entrevista a Miguel López Girón, San Cristóbal de Las Casas, 5 de febrero de 2018).

Ante mi pregunta acerca de la distribución de los campamentos mencionados, la mayoría instalados cerca de sus respectivas comunidades, Guadalupe (CDHFBC) considera que "en un momento en que está toda la situación de agresión, es correrse un poco, hacia donde podían” (Entrevista a Guadalupe Moshan, San Cristóbal de Las Casas, 7 de febrero de 2018). Así, la movilidad, en tanto escape o huida ante situaciones de fuerte violencia, puede plantearse aquí como un último intento de ejercer la agencia desde una posición de profunda inseguridad (Gill, Caletrío y Mason, 2014: 3). "Es el nivel de violencia que los hace que se queden, que continúen o que salgan por completo del lugar. Porque, por ejemplo, a Chalchihuitán, ahorita recordé también, por qué se mueven sólo a un lado... porque no tenían otras vías de salida, se cercó todo. Por ejemplo, Chenalhó cercó todas las salidas" (ídem).

De ese modo, Guadalupe se refiere a las opciones que se les han puesto en juego a la hora de refugiarse en dichos lugares. Resulta por ello de relevancia indagar en las características de los bloqueos, cierres forzados de caminos $\mathrm{y}$ accesos, pues ha sido esta combinación de desplazamiento e inmovilidad forzada la que trajo aparejada graves consecuencias en términos humanos y materiales: la proliferación de enfermedades, ${ }^{23}$ fallecimientos ${ }^{24}$ y pérdidas Xixim Tontic y 133 personas de Jolc’ante'tic (Cáritas, 2018).

${ }^{23}$ Según el Informe de la Brigada Médica, en Chalchihuitán, Chiapas (SADEC 2017) las enfermedades diagnosticadas han sido: respiratorias (46\% del total), Gastrointestinales (15\%), Trastornos de ansiedad (10\%), Padecimientos osteomusculares (6\%), Problemas relacionados con la mala alimentación (4\%), Infecciones de Vías Urinarias (3\%), Problemas de la piel (3\%), Otras (6\%), Sanos, entre estos 10 embarazadas (4\%).

${ }^{24}$ Las causas de los fallecimientos han sido: Neumonía: Maura Pérez Luna, 1 año 6 meses, falleció el 5 diciembre, localidad Pom. Mariano Pérez Aguilar, adulto mayor, falleció el 12 diciembre. María Domínguez Gómez, 57 ańos, falleció el 13 diciembre, localidad Pom. Hipotermia: Domingo Girón Luna, 70 años, falleció el 9 diciembre, localidad, Pom. Martín Girón Rodríguez, 80 años, localidad Canan Lumtic. 1 muerte fetal: Hija(o) de Catarina 
de cosechas y pertenencias. Como indica la Comisión Interamericana de Derechos humanos, luego del desplazamiento forzado en noviembre de 2017, "la falta de acceso al municipio habría desencadenado una "crisis humanitaria" ante la necesidad y urgencia de comida (maíz, frijol, maseca ${ }^{25}$, sal, azúcar), ropa para cubrirse del frío, utensilios de cocina y de limpieza, así como la necesidad de brigada médica" (CIDH, 2018: 2).

En el mapa a continuación señalamos con color rojo las ubicaciones aproximadas de los bloqueos de caminos -cerrados con troncos, zanjas y/o controles de grupos armados- que durante un mes mantuvieron en situación de aislamiento e inmovilidad forzada a las personas desplazadas.

Imagen IV: "Chalchihuitán: campamentos y bloqueos"

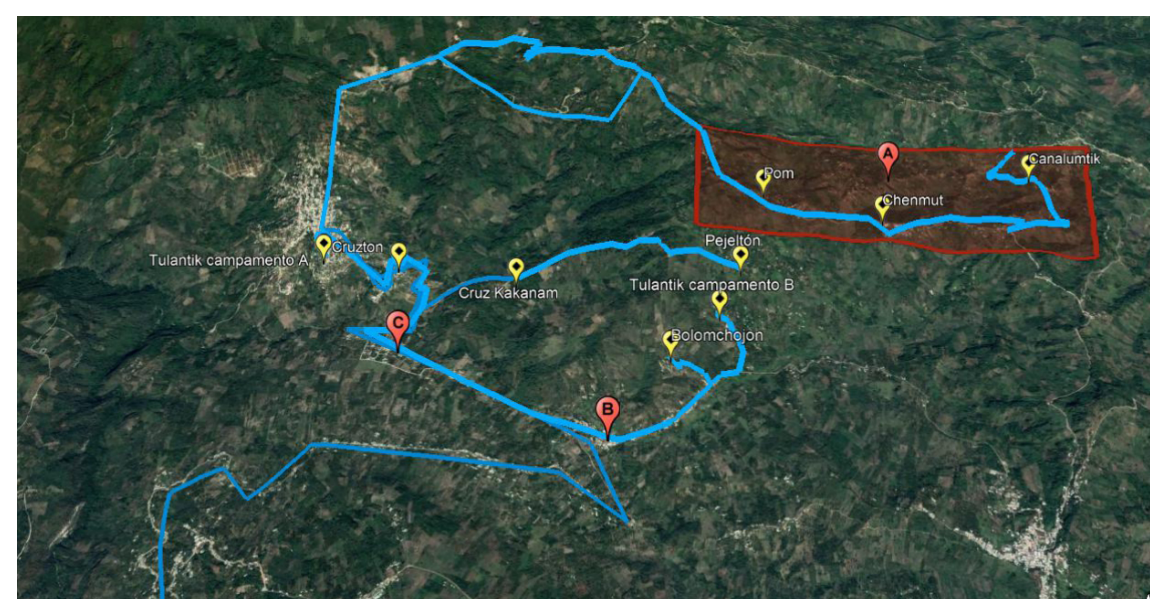

Fuente Elaboración de la autora y Miguel López Girón, 2019.

El bloqueo indicado con el punto "B" (Imagen IV), se encuentra también mencionado en la Medida Cautelar No 882-17 de la Comisión Interamericana de Derechos Humanos (CIDH, 2018): "en la Comunidad de Las Limas existiría

Sánchez Pérez, 15 de noviembre, localidad Tzomoñton. Diabetes descompensada: Marcelino Gómez López, adulto, padecía diabetes, presentó complicación, no pudo ser trasladado a San Cristóbal de Las Casas por bloqueo en carretera. Por arma de fuego: Samuel Luna Girón, adulto, falleció el 18 de octubre. Intoxicación por herbicida: Raymundo Luna Pérez, 17 años, falleció el 17 diciembre, localidad Chen Mut. Causa desconocida: Adriana de Jesús Pérez Pérez, 2 meses, falleció el 30 noviembre, localidad Pom. Recién nacida Ernestina Sánchez Pérez de Canan Lumtic (SADEC, 2017).

${ }^{25}$ Marca de harina de maíz. 
un bloqueo carretero donde fue abierta una zanja, [y] permanecen habitantes de Chenalhó presuntamente armados y encapuchados". Este bloqueo cierra la carretera entre los municipios de Chalchihuitán y Chenalhó, lo cual, a su vez, obstruye la conexión entre el primero y la ciudad de San Cristóbal de las Casas, es decir, lo desconecta de uno de los principales centros de la región, lo cual ha dificultado además la llegada de ayuda médica y asistencia a la zona.

Así, organizaciones como la brigada médica de Salud y Desarrollo Comunitario A.C. y la Casa de La Mujer, Ixim Antsetic A.C. denunciaron que les prohibieron el paso cuando iban a brindar asistencia a las personas desplazadas y que, por lo tanto, tuvieron que tomar un camino alternativo para llegar a los campamentos, pasando por el municipio de Simojovel y demorando siete horas en llegar (CDHFBC, 2017: 1), en lugar de la hora y media que es lo que dura el trayecto por la carretera principal.

Sinuoso y de montaña, con elevaciones que llegan a los 2554 metros, este camino, de alrededor de una hora y media desde San Cristóbal de Las Casas, fue bloqueado en varias oportunidades. Según Miguel, ellos lograron pasar por

Imagen V: "Primer tramo del camino desde San Cristóbal de las Casas hacia Chalchihuitán (carretera San Cristóbal-Pantelhó)"

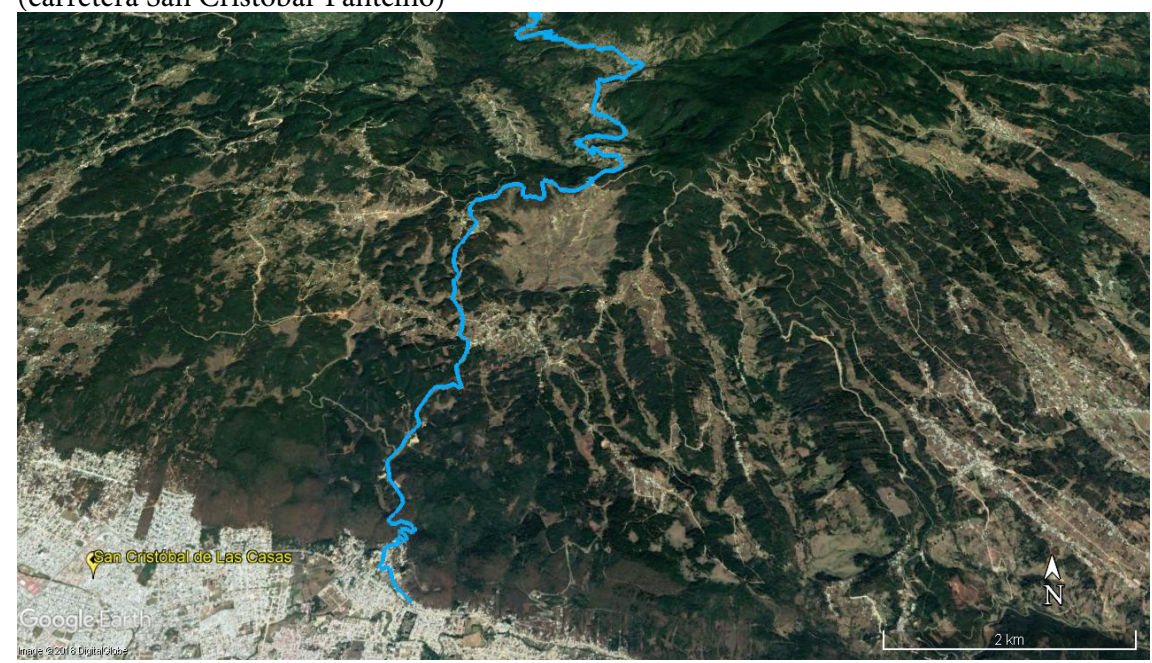

Fuente: Elaboración de la autora y Miguel López Girón, 2019.

tratarse de una organización de ayuda humanitaria internacional (MSF): "me tocó el bloqueo, las personas dijeron que nos dejaran pasar porque estamos en 
apoyo a toda la comunidad" (Entrevista a Miguel López Girón, San Cristóbal de Las Casas, 30 de enero de 2018).

Los bloqueos en el tramo entre las comunidades Pom y Canalumtic (punto "A" en Imagen IV) y en Tzomoltón (punto " $\mathrm{C}$ " en Imagen IV) restringen la movilidad entre las comunidades cercanas al área de conflicto y cierran conexiones con la cabecera municipal de Chalchihuitán. Su ubicación es mencionada además en artículos de prensa (Marroquín, 2017; Gutiérrez, 2017; Mandujano, 2017) y en un Acta de Acuerdo del municipio de Chenalhó:

Con el objetivo de generar las situaciones a efecto de que el Tribunal Unitario Agrario Distrito 3 con sede en Tuxtla Gutiérrez, Chiapas, emita la Sentencia Definitiva al conflicto agrario entre los municipios, toda vez que el gobierno del estado tiene conocimiento que desde el pasado 14 de noviembre del año en curso, un grupo de comuneros respaldados por 11 comunidades bloquearon las vías de acceso al municipio de Chalchihuitán, en los siguientes puntos: Tsomoltón, Pom, Canalumtic. En ese contexto, solicitó se busque las alternativas de solución para que sean liberadas las vías obstruidas ("Acta de Acuerdo del Ayuntamiento Municipal de Chenalhó", 5 de diciembre de 2017:2).

La fecha indicada en el acta como inicio de estos bloqueos, el 14 de noviembre de 2017, corresponde al día posterior a que las agresiones armadas dieran lugar al desplazamiento forzado. Allí se refieren a quienes los han llevado a cabo como "un grupo de comuneros respaldados por once comunidades", ya que las autoridades del municipio de Chenalhó sostienen que estas acciones forman parte de una lucha legítima por recuperar tierras que le pertenecen. Acerca de ello, cabe señalar, se trata de grupos que han sido caracterizados como de corte paramilitar e identificados como ejecutores del desplazamiento forzado en los márgenes del conflicto (García Núñez y Cruz Rueda, 2019: 36).

Así, en la misma Acta de Acuerdo antes citada, agregan que:

[...] se aclara que en caso de que el Tribunal Unitario Agrario no emita la resolución definitiva y a favor del municipio de Chenalhó, se llevarán a cabo medidas drásticas en contra de los comuneros del municipio de Chalchihuitán, 
hasta recuperar las tierras que ancestralmente pertenecen al municipio de Chenalhó (Ídem).

El compromiso que las autoridades de Chenalhó expresan en el Acta anterior, respecto a liberar las vías señaladas, es provisorio y condicional, sujeto a la resolución del Tribunal Unitario Agrario. Además, se trataría de un desbloqueo parcial, sólo para el tránsito peatonal, no vehicular. ${ }^{26}$

\section{Conclusiones}

En el primer apartado de este artículo presento los antecedentes del conflicto limítrofe entre los municipios de Chenalhó y Chalchihuitán mediante sus registros oficiales, formales y escritos, los cuales deben ser comprendidos como una de las dimensiones del mismo, que no lo abarcan en su extensión y complejidad, quedando pendiente profundizar la investigación en sus dimensiones culturales y simbólicas.

Explico entonces el establecimiento formal del conflicto, en la década de 1970, con la intervención de la Secretaría de la Reforma Agraria en el proceso de reconocimiento de la propiedad sobre los bienes comunales de ambos municipios. A partir ello, describo el modo en que se contraponen diferentes concepciones acerca de los límites territoriales y la manera en que se ponen en juego los documentos estatales para intentar legitimar la marcación limítrofe de cada una de las partes. Así, mientras Chalchihuitán se basa en una "línea artificial" marcada mediante la resolución presidencial del 29 de agosto de 1975 (y sus correspondientes actas de posesión y deslinde), las autoridades de Chenalhó argumentan que debe respetarse la "colindancia natural" del río, de tiempo inmemorial, y consideran legítimo el acuerdo celebrado por ambos municipios en el año 1976.

El artículo da cuenta del modo en que las prácticas de marcación limítrofe despliegan su conflictividad tanto en el ámbito jurídico formal -documentos escritos vinculados al reconocimiento y titulación de bienes comunales, resoluciones presidenciales, actas de posesión y deslinde, juicios agrarioscomo en la materialidad del territorio, mediante la instalación de mojones, construcción y derribamiento de muros y prácticas de violencia armada. En

\footnotetext{
${ }^{26}$ Según la Comisión Estatal de los Derechos Humanos (CEDH, 2018) el día 15 de diciembre de 2017 el tramo fue liberado por completo luego del fallo del Tribunal Unitario Agrario (TUA) a favor de Chenalhó.
} 
su punto más cruento, estás prácticas provocaron el desplazamiento forzado de más de 5000 mil personas, cuyos efectos en términos materiales y humanos describo en el último apartado del artículo.

$\mathrm{Al}$ respecto, es posible plantear a modo de síntesis y conclusión que, a partir de una combinación particular de movimientos (de huida o escape) y restricciones (cierre de caminos y conexiones), los procesos de (in)movilidad forzada en la zona de conflicto derivaron en la construcción de campamentos (refugios auto-establecidos) inmersos en un contexto de crisis humanitaria -en tanto situación de emergencia en que la vida, la salud y el bienestar de las personas desplazadas se ven amenazadas- y de (des)territorialización precaria, en la medida en que la violencia ha traído como efectos una fuerte inestabilidad e inseguridad socioespacial, movilidades sin dirección definida e inmovilidades sin un efectivo control territorial.

Dejo pendiente para futuros trabajos el abordaje de estas (in)movilidades forzadas desde una perspectiva de más largo aliento.

\section{Bibliografía}

Agier, Michel (2012) "El biopoder a prueba de sus formas sensibles" en Politica y Sociedad. Vol. 49, Núm. 3. pp. 487-495.

Arana Cedeño, Marcos y María Teresa Del Riego (2012) Estudio sobre los desplazados por el conflicto armado en Chiapas. México, México: Programa conjunto por una cultura de paz.

Cámara de Diputados del Congreso de la Unión, Boletín núm. 4219 (2017), "Diputados aprueban sustituir denominación de Secretaría de la Reforma Agraria por la de Desarrollo Agrario, Territorial y Urbano", 17 de octubre de 2019. Disponible en: <http://www5.diputados.gob. mx/index.php/esl/Comunicacion/Boletines/2017/Octubre/17/4219Diputados-aprueban-sustituir-denominacion-de-Secretaria-dela-Reforma-Agraria-por-la-de-Desarrollo-Agrario-Territorial-yUrbano> [consultado 14 de diciembre de 2019].

Cámara de Diputados del Congreso de la Unión. Comisión de Reforma 
Agraria de la Cámara (2009) "Puntos de acuerdo por los que se exhorta a la Secretaría de la Reforma Agraria a continuar atendiendo el conflicto agrario entre Chenalhó y Chalchihuitán en Chiapas privilegiando el diálogo y la conciliación", 3 de marzo de 2009. Disponible en: <http:// sil.gobernacion.gob.mx/Archivos/Documentos/2009/04/asun_2569 367_20090428_1240957061.pdf> [Consultado 15 de diciembre de 2019].

Cáritas de San Cristóbal (2018) "Informe atención a desplazados de Chalchihuitán y Chenalhó" en Cáritas San Cristóbal, 17 de abril de 2018. Disponible en: http://www.caritasancristobal.org/informeatencion-a-desplazados-chalchihuitan-chenalho/ [consultado el 25 de noviembre de 2018].

Centro de Derechos Humanos Fray Bartolomé de Las Casas (CDHFBC) (2017) Informe de Brigada Médica en Chalchihuitán. San Cristóbal de Las Casas, Chiapas: Centro de Derechos Humanos Fray Bartolomé de Las Casas. Disponible en: https://frayba.org.mx/informe-de-brigadamedica-en-chalchihuitan/ [consultado el 17 de diciembre de 2019].

Centro de Derechos Humanos Fray Bartolomé de Las Casas (CDHFBC) (2018) Acción Urgente: En riesgo la vida de familias desplazadasretornadas en Chalchihuitán. San Cristóbal de Las Casas, Chiapas: Centro de Derechos Humanos Fray Bartolomé de Las Casas. 4 de enero 2018. Disponible en: https://frayba.org.mx/accion-urgenteen-riesgo-la-vida-y-la-integridad-de-centenares-familias-desplazadasretornadas-en-chalchihuitan/ consultado el 16 de diciembre de 2019].

Centro de Derechos Humanos Fray Bartolomé de Las Casas (CDHFBC) (2019). "Equipo zona Altos" Disponible en: <https://frayba.org.mx/ zona-altos/> [consultado 21 de diciembre de 2019].

Chamberlin, Michael (2013) "El desplazamiento forzado como estrategia de guerra en Chiapas" en Torrens, O. (Coord.) El desplazamiento interno forzado en México. Un acercamiento para su reflexión y análisis. México: CIESAS, Colegio de Sonora, Senado de la República, LXII Legislatura, Comisión de Biblioteca y Asuntos Editoriales.

Comisión Estatal de los Derechos Humanos (CEDH) (2017) La CEDH constata levantamiento de bloqueos entre Chalchihuitán y Chenalhó. Chiapas: Sala de Prensa, Comisión Estatal de Derechos Humanos. 
Disponible en: https://www.cedh-chiapas.org/portal/index.php/ conocenos/promocion/sala-de-prensa/boletines-2017/866-lacedh-constata-levantamiento-de-bloqueos-entre-chalchihuitan-ychenalho.html [consultado el 15 de diciembre de 2019].

Comisión Interamericana de Derechos Humanos (CIDH) (2018) "Comunidades indígenas tsotsiles de Chalchihuitán y Chenalhó respecto de México" Medida Cautelar. Núm. 882-17, Resolución 15/2018, 24 de febrero de 2018. Organización de los Estados Americanos, Comisión Interamericana de Derechos Humanos.

Comisión Mexicana de Defensa y Promoción de los Derechos Humanos (CMDPDH) (2019) Episodios de Desplazamiento Interno Forzado masivo en México. Informe 2018. México: Comisión Mexicana de Defensa y Promoción de los Derechos Humanos. También disponible en <http://www.cmdpdh.org/publicaciones-pdf/cmdpdh-episodiosde-desplazamiento-interno-forzado-en-mexico-informe-2018.pdf>

Coraza de los Santos, Enrique, (2018) "Movilidades forzadas. Cuando moverse es una estrategia de vida" en Revista Ecofronteras. Vol. 22, No 62. pp. 18-20.

Diario Oficial de la Federación (DOF) (1975) 29 agosto 1975, p.16.

Diario Oficial de la Federación (DOF) (1975) 6 octubre 1975, p. 17.

García Núñez, Eugenio y Elisa Cruz Rueda, (2019) “Chalchihuitán y Chenalhó: del conflicto agrario a la gestión humanitaria” en Ortelli, Paola y Lauriano E. Rodríguez Ortiz (coords.) Experiencias contemporáneas de participación politica y ciudadana en México y Colombia. San Cristóbal de Las Casas: Licenciatura en Gestión y Autodesarrollo IndígenaUniversidad Autónoma de Chiapas; Programa de Fortalecimiento de la Calidad Educativa-Secretaría de Educación Pública. pp. 11-46.

Gill, Nick; Caletrío, Javier y Victoria Mason (2014) "Introduction: Mobilities and Forced Migration” en Gill, Nick; Caletrío, Javier y Victoria Mason (eds.), Mobilities and Forced Migration. London: Routledge.

Gobierno de México (2020) "Lenguas indígenas. Tsotsil" en Sistema de información Cultural Disponible en: <http://sic.gob.mx/ficha. php?table=inali_li\&table_id=51\&disciplina $=>\quad\left[\begin{array}{ccc}\text { consultado } & 9 & \text { de }\end{array}\right.$ enero de 2019].

Gutiérrez, Leonardo (2017) "Fallecen por el frío cuatro niños, hijos de padres 
desplazados en Chalchihuitán” Ultimatum Chiapas, 11 de diciembre 2017. Disponible en: <http://ultimatumchiapas.com/fallecen-frio4-ninos-hijos-padres-desplazados-chalchihuitan/> [Consultado 8 de noviembre de 2018].

Grupo Multidisciplinario (2010 ms.) Informe que se rinde al Comité Técnico para la atención del conflicto de limites entre las comunidades de San Pedro Chenalhó y San Pablo Chalchihuitán, Chiapas, México. (Informe inédito). San Cristóbal de las Casas. Marzo de 2010.

Haesbaert, Rogerio (2011) El mito de la desterritorialización. Del fin de los territorios a la multiterritorialidad. México: Siglo XXI.

Henríquez, Elio (2008) “Asesinan a machetazos a tzotzil" La Jornada, 8 de abril de 2008. Disponible en: <ttp://www.jornada.com.mx/2008/04/08/ index.php?section=estados $\&$ article $=034 \mathrm{n} 1$ est $>$ [Consultado 24 de octubre de 2018].

Henríquez, Elio (2014) "Destruyen mojoneras de tierras en disputa" La Jornada, 16 de mayo de 2014. Disponible en: https://www.jornada. com.mx/2014/05/16/estados/032n5est [Consultado 15 de diciembre de 2019].

Henríquez, Elio (2015) “Desplazados, 300 indígenas de Chenalhó, por conflicto agrario. En Chalchihuitán les imputan asesinato" La Jornada, 12 de julio de 2015. Disponible en: http://www.jornada. com.mx/2015/07/12/estados/023n2est [Consultado 25 de octubre de 2018].

Henríquez, Elio (2017) “Tribunal Unitario Agrario ordena restituir 365 hectáreas a Chenalhó" en La Jornada. 14 de diciembre de 2017. Disponible en: <http://www.jornada.com.mx/2017/12/14/ politica/007n1 pol> [Consultado 15 de diciembre de 2019].

Instituto Nacional de Estadística, Geografía e Informática (INEGI) (2010) Censo de Población y Vivienda 2010.

Jiménez Huerta, Edith R. y Ayala, María de la Luz, (2015) "Los ejidos y comunidades indígenas ante la expansión de Guadalajara, 1920-2000" en Olivera, G. (coord.), La urbanización social y privada del ejido: ensayos sobre la dualidad del desarrollo urbano en México. Cuernavaca: Centro Regional de Investigaciones Multidisciplinarias, Universidad Nacional Autónoma de México. pp. 103-149. 
Mandujano, Isaín (2017) “ONG exhibe el terror de los desplazados en Chiapas” en Proceso. Disponible en: <https://www.proceso.com. $m x / 514980 /$ ong-exhibe-el-terror-de-los-desplazados-en-chiapas $>$ [Consultado $8 \mathrm{de}$ noviembre de 2018].

Marroquín, A. (2018) “Chalchihuitán está secuestrado por Chenalhó, habitantes huyen" Aquí Noticias, 24 de noviembre de 2017, Disponible en: http://aquinoticias.mx/chalchihuitan-esta-secuestrado-chenalhohabitantes-huyen/ [Consultado 8 de noviembre de 2018].

Martínez Velasco Germán, (2005) “Conflicto étnico y migraciones forzadas en Chiapas" en Revista Politica y Cultura. No. 23. México: UAMXochimilco. pp. 199-201.

Médicos Sin Fronteras (MSF) (sin fecha) "Quiénes somos” Disponible en: $<$ https://www.msf.org.ar/conocenos/quienes-somos> [Consultado 20 de diciembre de 2019].

Médicos Sin Fronteras (MSF) (2018) "México: atención médica a los desplazados en Chiapas" en Médicos Sin Fronteras. 22 de enero de 2018. Disponible en: https://www.msf.org.ar/actualidad/mexicoatencion-medica-desplazados-chiapas [consultado el 15 de diciembre de 2019].

Organización de Las Naciones Unidas (ONU) (2020) Sistema de Las Naciones Unidas en Perú, "Desastres y crisis humanitarias". Disponible en: <http://onu.org.pe/temas/desastres-ycrisis-humanitarias/> [Consultado 14 de abril de 2020].

Periódico Oficial del Estado de Chiapas Núm. 355, 22 de Febrero de 2012. "Ley para la Prevención y Atención del Desplazamiento Interno en el Estado de Chiapas” Decreto No 158, 22 de Febrero de 2012.

Poder Judicial del Estado de Chiapas (2017) "Acta de posesión y deslinde con fecha 1 de diciembre de 1980" en Boletín del Poder Judicial del Estado de Chiapas. Sintesis informativa. 15 de diciembre de 2017: p. 15.

Programa de las Naciones Unidas para el Desarrollo (PNUD), et al. (2016) "Plan Municipal para la Reducción de Vulnerabilidades y Riesgos. Municipio de Chenalhó, Chiapas", marzo 2016. Disponible en: $<$ https://proteccioncivil.chiapas.gob.mx/documentos/2016/planesmunicipales-pnud-2016/chenalho-plan-rrd-2016.pdf> [Consultado 17 de abril de 2020]. 
Programa de las Naciones Unidas para el Desarrollo (PNUD), et al. (2016) "Plan Municipal para la Reducción de Vulnerabilidades y Riesgos. Municipio de Chalchihuitán, Chiapas”, 2016. Disponible en <https:// proteccioncivil.chiapas.gob.mx/documentos/2016/planes-municipalespnud-2016/chalchihuitan-plan-rrd-2016.pdf> [consultado 17 de abril de 2020]

Reyes Ramos, María Eugenia (2004) "Política agraria en Chiapas: atención a focos rojos" en Estudios Agrarios. No. 26. México: Procuraduría Agraria. pp. 55-93.

Rivera Farfán, Carolina (2013) “Creencias y prácticas religiosas censuradas. Expulsión de evangélicos indígenas por cambio de adscripción religiosa" en Torrens, O. (Coord.), El desplazamiento interno forzado en México. Un acercamiento para su reflexión y análisis. México: Centro de Investigaciones y Estudios Superiores en Antropología Social, Colegio de Sonora, Senado de la República, LXII Legislatura, Comisión de Biblioteca y Asuntos Editoriales.

Salud y Desarrollo Comunitario A.C. (SADEC) (2017) Informe de Brigada Médica en Chalchihuitán, Chiapas. Diciembre 2017. Disponible en https://frayba.org.mx/wp-content/uploads/2017/12/Informe-finalBrigada-m\%C3\%A9dica-Chalchihuit\%C3\%A1n-SDC-CAM.pdf. [Consultado el 15 de diciembre de 2019]

Santiago Rodríguez, Guillermo Rafael (2017) Con punto de acuerdo, relativo a la implantación de medidas y acciones a fin de dar pronta solución al conflicto entre las comunidades de Chalchibuitán y Chenalhó, y garantizar el apoyo para los desplazados por la violencia en Los Altos, a cargo del diputado Guillermo Rafael Santiago Rodríguez, del grupo parlamentario de Morena. México: LXIII Legislatura de la honorable Cámara de Diputados, Palacio Legislativo de San Lázaro. 12 de diciembre de 2017. Disponible en: http://sil.gobernacion.gob.mx/ Archivos/Documentos/2017/12/asun_3644195_20171212_151311 2824.pdf [Consultado el 15 de diciembre de 2019].

Sheseña, Alejandro (2014) "El papel de las cuevas en las guerras de los antiguos mayas" en Itinerarios Revista de estudios lingüisticos, literarios, históricos y antropológicos. Vol. 19. 


\section{Documentos judiciales}

Décimo Tercer Tribunal Colegiado en Materia Administrativa del Primer Circuito, (2005) "Solicitud de Ejercicio de la Facultad de Atracción 19/2004-SS”. 14 enero 2005.

Décimo Tribunal Colegiado en Materia Administrativa, (2004) Amparo Directo, 39/2004-550.

Tribunal Unitario Agrario (TUA) de Distrito No 3 (2000) Juicio No 995/2000. Tribunal Unitario Agrario (TUA), (2003) Recurso de Revisión 405/200303.

\section{Entrevistas}

Entrevista a Guadalupe Moshan, San Cristóbal de Las Casas, 5 de febrero de 2018.

Entrevista a Miguel López Girón, San Cristóbal de Las Casas, 5 de febrero de 2018.

Entrevista a Guadalupe Moshan, San Cristóbal de Las Casas, 7 de febrero de 2018.

Entrevista a Miguel López Girón, San Cristóbal de Las Casas, 30 de enero de 2018. 